\title{
CATETERISMO RETRÓGRADO EM NEURO-RADIOLOGIA
}

\author{
SÉrgio F. RAUPP * \\ PaUlo A. Bolli **
}

O diagnóstico radiológico das lesões vasculares cerebrais ganhou em profundidade com a introdução de técnicas que permitem visibilizar as origens extracranianas da rêde vascular encefálica. Diversas técnicas para visualização dos vasos aorto-cervicais foram exaustivamente experimentadas 1, 2, 5, 7, 9, 13, 14. Em nosso Serviço, após experimentações continuadas, com períodos alternados de entusiasmos e desilusões, fomos cristalizando nossa conduta e codificando a rotina das técnicas utilizando o cateterismo retrógrado (Seldinger ${ }^{12}$ ), com cateteres de Odman-Ledin ${ }^{11,12}$. O presente trabalho visa a expor as indicações e nosso modo de executar o referido método, assinalando as complicações mais freqüentemente observadas.

INDICAÇõEs - Utilizamos o cateterismo retrógrado: 1) no estudo de casos de claudicação cerebral intermitente, nos quais são procuradas lesōes arterioscleróticas nas porções extracranianas dos vasos; 2) na angiografia vértebro-basilar quando utilizada no diagnóstico de rotina; 3) na prática de angiografias vẻrtebro-basilares transoperatórias; 4) para a angiografia seletiva da carótida interna ou externa, ou ambas, nos casos de tumores da base de crânio e da face, ou naqueles em que se suspeite a existência de pediculos meníngeos (meningeomas); 5) nos casos de hemorragias cerebrais sem sinais clínicos localizadores, nos quais é possível prever a necessidade da investigação dos dois sistemas carotídeos e do sistema vértebrobasilar; 6) no estudo das vasculopatias medulares ${ }^{6}$.

Método - Seguimos as regras especificas do cateterismo retrógrado segundo Seldinger ${ }^{12},{ }^{13},{ }^{15}$. O paciente é submetido de preferência à sedação anestésica com neuroleptanalgesia tipo II. Usamos a artéria femoral direita como via de acesso quando o pulso é satisfatório, tendo como opçōes a artéria femoral contralateral, a artéria axilar e a artéria umeral. Efetuamos sistemàticamente a punção percutânea do vaso, com pequena incisão na pele $(1$ a $3 \mathrm{~cm})$; jamais realizamos dissecção do vaso com arteriotomia, seja qual fôr a artéria visada. O calibre do catéter varia em função da meta a atingir: $a$ ) aortografia - marron ou amarelo (Kifa); b) vasos supra-aórticos - verde ou vermelho (Kifa). No cateterismo seletivo, quando usamos a artéria femoral como via de acesso, é fundamental que a aortografia seja feita prèviamente pois isso permite conhecer a anatomia dos vasos supra-aórticos.

O uso do intensificador de imagem é de enorme valia, em especial no cateterismo seletivo de pacientes excessivamente obesos ainda que, na grande maioria dos casos, a fluoroscopia, criteriosamente usada, permita levar a cabo as investigaçōes.

Nas injeções da aorta ascendente usamos o injetor de Gidlung e, no cateterismo de vaso de menor calibre, a injeção manual.

Instituto de Neurocirurgia de Pôrto Aleọre: * Neuro-radiologista; ** Médico residente. 
COMPlicaçōes - Em análise de 11.402 casos Lang ${ }^{8}$ refere mortalidade de 0,06\%; afora isto, as complicaçóes observadas se devem a defeito de técnica ou a falta de experiência do executor.

Espasmos arteriais ocorrem devido à repetição de punçōes ou ao avanço forçado da guia ou do catéter. Não acreditamos na eficiência de medicação auxiliar. Quanto menor fôr o calibre do vaso, maior será a incidência desta complicação.

Falsa via - perfuração da parede do vaso. Em nossa casuística tivemos esta complicação uma única vez tendo sido perfurada a artéria ilíaca. O mau estado da guia metálica foi certamente o causador do acidente. Material em perfeitas condições é de capital importância.

Hematomas têm como causas as puncōes defeituosas, o excessivo manuseio do vaso ou a falta de compressāo após o exame. A heparinização prévia do paciente não impede o procedimento.

Tromboses arteriais ocorrem principalmente em pacientes diabéticos, hipertensos e arterioscleróticos. Sob êste aspecto, as artérias do membro superior apresentam melhor circulação colateral que a artéria femoral, razão pela qual êste acidente é menos grave no território braquial. A diminuição ou desaparecimento do pulso sem sinais de isquemia não é significativo de trombose. Em tal situação as atitudes cabiveis são o uso de anticoagulantes, o bloqueio do simpático e a intervenção cirúrgica. Oclusão vascular não ocorrerá quando evitarmos: a) a utilização de catéter excessivamente calibroso em relação à artéria; b) duração demasiada do exame; c) excessiva compressão após a injeção do contraste (compressão suave e continuada).

\section{S U M M A R Y}

\section{Retrognade catheterization in Neuro-radiology.}

The bases and technical cares for the neuro-radiological study of the aorto-cervical and spinal vessels employing the retrograde catheterization according to Seldinger technic and with the Odman-Ledin catheters are reported. The authors recommend type II neuroleptanalgesia as anesthesical sedative and the use of percutaneous punction of the femoral artery or, by choice, of the axilar or humeral artery. For the selective catheterization by femoral via, they make previously an aortography, in order to know the anatomy of the supra-aortic vessels, with control through the image-intensifier or fluoroscopy. They employ manual injection for the study of the supra-aortic vessels and a Gidlung injector for the contrast of the ascending aorta. Complications are discussed.

\section{R E F E R E N I A S}

1. AMPLATZ, K. - Percutaneous arterial catheterization and its application. Amer. J. Roentgen. 87:265, 1962. 2. AMPLATZ, K. \& HARNER, R. - A new subclavian artery catheterization technic. Radiology 78:963, 1962. 3. BETOULIERER, P.; PELISSIER, M. \& MINRAU, R. - L'aortographie retrograde par voie femorale percutanée. Presse Med. 65:2159, 1957. 4. MOIJSEN, E. \& FEINSTEIN, G. I. - Arteriographic catheterization techniques. Amer. J. Roentgen. 85:1037, 1961. 5. CURRY, Y. S. \& HOWLAND, W. Y. - Arteriography: Principles and Techniques. W. B. Saunders, Philadelphia, 1966. 6. DI CHIRO, J.; DOPPMAN, F. \& OMMAYA, A. K. - Selective arteriovenous aneurysms of spinal cord. Radio- 
logy 88:1065, 1967. 7. GENSINI, G. \& ECLER, A. - Percutaneous aortocerebral angiography. Radiology $75.885,1960$. 8. LANG, E. $\mathbb{K}$. - A survey of the complications of percutaneous retrograde arteriography. Seldinger technic. Radiology 81:257, 1963. 9. NEWTON, T. H. - The axillary artery approach to arteriography of the aorta and its branches. Amer. J. Roentgen. 89:275, 1963.10. ODMAN, P. - Thoracic aortography by means of a radiopaque polyethylene catheter inserted percutaneously. Acta Radiol. 45:117, 1956. 11. ODMAN, P. - Radiopaque polyethylene catheter. Acta Radiol. 52:52, 1959. 12. SELDINGER, S. I. - Catheter replacement of the needle in percutaneous arteriography. A new technic. Acta Radiol. 39:318, 1953. 13. TOOLE, Y. F. - Special Techniques for Neurologic Diagnosis. F. A. Davis Co., Philadelphia, 1969. 14. WESTCOTT, Y. S.; CHYM, K. Y. \& STEIMBERG, I. - Percutaneous transfemoral selective arteriography of the brachiocephalic vessels. Amer. J. Roentgen. 90:554, 1963. 15. ZIMMERMANN, H. A. - El Cateterismo Cardiovascular. Editorial Cientifico-Medica, Barcelona, 1969.

\title{
ANGIOGRAFIA VÉRTEBRO-BASILAR POR CATETERISMO RETRÓGRADO
}

\author{
SÉRgIo F. RAUPP * \\ Luiz ReNato Mello **
}

A angiografia vértebro-basilar foi realizada pela primeira vez em 1933 por Egaz Moniz mediante dissecção da artéria subclávia. Nos 36 anos que desde então decorreram foram propostas técnicas as mais diversas para resolver o problema dos vasos de pequeno calibe. Podemos agrupá-las em três classes:

$A$ - Injeção por punção direta ${ }^{1},{ }^{2},{ }^{6},{ }^{8},{ }^{12},{ }^{13}$ - Em 1937, Sjöqvist dissecou o vaso na entrada do canal transversário. Takahashi (1940) descreveu técnica com reparos anatômicos para punção direta na primeira porção da artéria. Olivecrona (1941) desnudou a artéria vertebral em sua origem. Seis anos mais tarde, Lindgren preconizou a punção direta percutânea, por via anterior, nos segmentos superiores do canal transversário, técnica que foi modificada, mais tarde, por Sjögren que sugeriu introdução da agulha pela linha mediana. Em 1954, Maslowski, realizou a punção direta, percutânea, por via sub-occípito-atlóidea.

$B$ - Injeção contra-corrente ${ }^{1},{ }^{2},{ }^{10},{ }^{12},{ }^{13}$ - Em 1937, Shimidzu, mediante punçāo percutânea da subclávia, injetou contraste contra a corrente sangüinea, fazendo compressão do vaso a montante. Engeset (1948) puncionou a carótida primitiva e comprimiu o vaso acima da punção ocasionando, à injeção de contraste, refluxo na subclávia e enchimento da artéria vertebral. Gould (1955) empregou injeção retrógrada, via artéria umeral. Em 1960, Pouyanne e col,, por via subclávia, puncionaram a artéria do mesmo nome, fazendo injeção contra-corrente. Záclis (1964) utiliza injeção de uma das carótidas concomitantemente à hipotensão sistêmica por hiperpressão pulmonar, acarretando refluxo de contraste ao sistema vértebro-basilar.

$C$ - Cateterização do vaso ${ }^{4},{ }^{7},{ }^{9},{ }^{11},{ }^{12}$ - Inicalmente Radner, em 1947, cateterizou a artéria vertebral através da artéria radial. Puncionando a artéria vertebral, Jaeger e Whiteley (1955), introduziram um cateter na luz do vaso. Lindgren, em 
1956, preconizou o cateterismo via artéria femoral quando falhe a punção direta. Amplatz (1962) sugeriu cateterismo da vertebral via artéria subclávia. Roy, no ano seguinte, indicou o cateterismo via artéria axilar.

Em nosso Serviço, com excessão das técnicas precedidas de dissecção do vaso, tentamos tôdas as outras adquirindo razoável experiência que nos capacita a crítica serena.

Nos procedimentos por punção direta lembramos as palavras de Ruggiero, citado por M. David ${ }^{2}$ : "Le gros inconvénient de la methode percutanée est la difficulté d'assurer le mantien de l'aiguille dans l'artére où, surtout quand le calibre de celle-ci est très mince, elle ne peut etre cathéterisée comme les artères carotides". Acrescente-se o fato de que uma das artérias ve itebrais pode se mostrar muito reduzida de calibre, não tendo o técnico condições de saber prèviamente qual a maior responsável pelo fluxo vértebro-basilar. Como conseqüência, as tentativas bilaterais são freqüentes, aumentando o desconfôrto do paciente. As injeções contra-corrente dão bons resultados entre $60-70 \%$ dos casos, ainda que o uso sistemático do intensificador de imagem permita caminhar em terreno relativamente firme. O enchimento retrógrado obtido mediante hiperpressão pulmonar ${ }^{14}$ nem sempre é cositivo. Além disso, peca contra uma das regras fundamentais da moderna neuro-radiologia qual seja a da seletividade, constrastando mais do que o desejado e fornecendo imagens parasitas. As técnicas de punção da subc'ávia podem acarretar pneumotórax, sendo o vaso pouco acessível à compressão.

Indiscutivelmente, dos procedimentos propostos, o que melhores condições reune no momento é o cateterismo retrógrado (Seldinger), com cateter de Odman-Ledin. Este método apresenta vantagens pois: a) punciona-se, como regra, um vaso de grande calibre, fixo e superficial (artéria femoral); $b$ ) pela aortografia ou injeções de pequenas quantidades de contraste identifica-se a artéria vertebral de maior calibre; $c$ ) existe a possibilidade de estudar o sistema desde o vaso nutridor (subclávia), até os mais finos ramos intracranianos (fig. 1); d) não se observam fenômenos vasomotores (espasmr:; ou artefatos por defeito de punção, que prejudiquem os clichês obtidos (fig. 1); e) o exame proposto sempre é realizado satisfatòriamente; $f$ ) rapidez de execução ( $30 \mathrm{~min}$. de "pele a pele") quando o procedimento é encarado como rotina e não como excessão.

Sòmente usamos as artérias axilares ou umerais como via de acesso nos casos em que o desenrolamento aórtico, da artéria inominada ou da subclávia, é acentuado, impedindo a progressão do catéter. Nos indivíduos jovens, com vasos em estado satisfatório, colocamos o catéter na luz da artéria vertebral. Em doentes arterioscleróticos ou com estenose do óstium, a injeção é feita ao nível da implantação na artéria subclávia.

Pelas razões expostas, em nosso Serviço, a exemplo do que ocorreu em outros $7,12,18$, as técnicas de angiografia vértebro-basilar por punção direta ou injeçōes contra-corrente foram definitivamente abandonadas. 

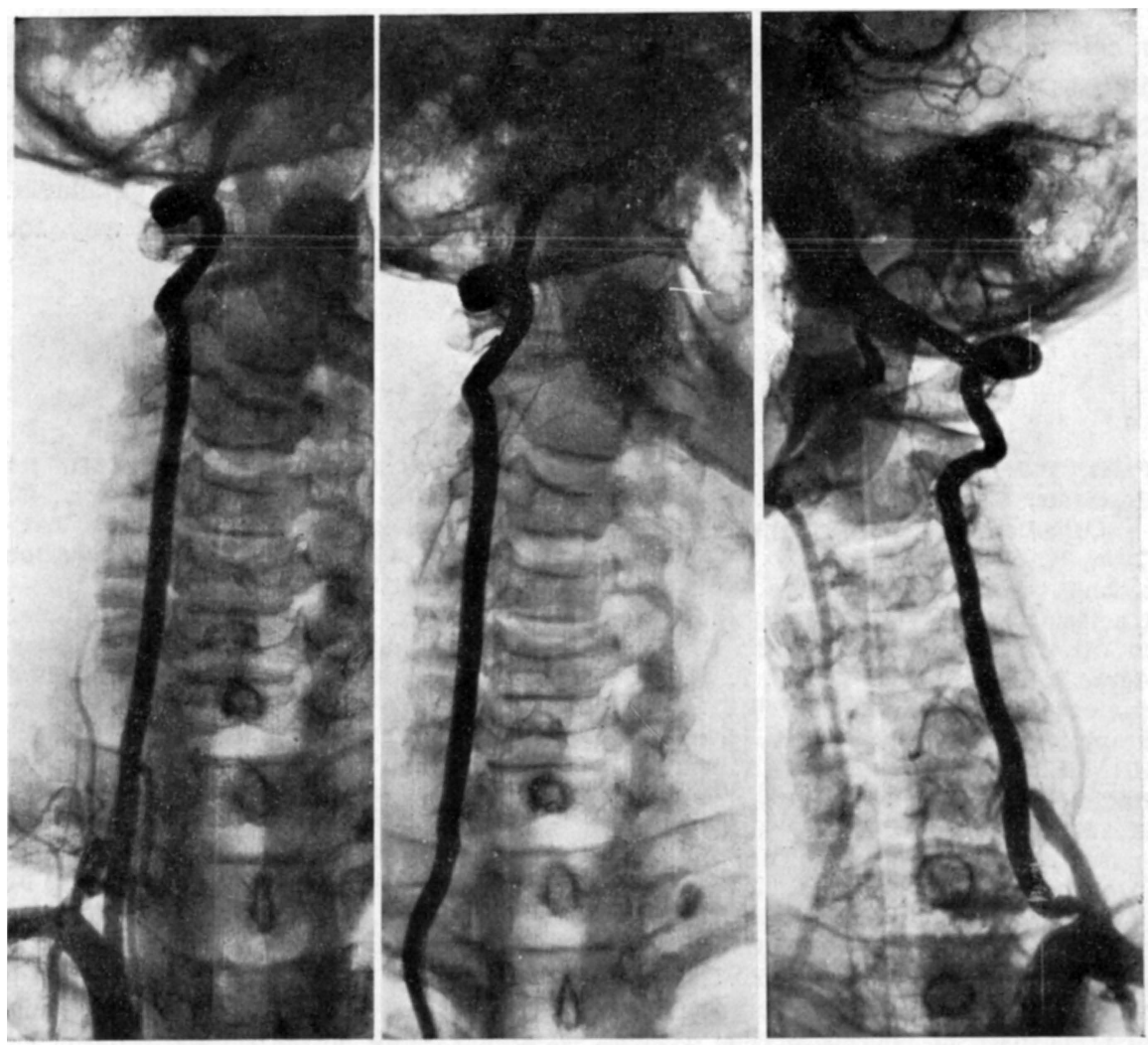

Fig. 1 - Angiografias vértebro-basilares por cateterismo retrógrado. Em A, cateterismo via artéria femoral direita estando o cateter colocado na artéria subclávia direita ao nivel da artéria vertebral do mesmo lado. $E m B$, mesmo caso que em $A$ estando o catéter introduzido na artéria vertebral, em seu segmento inferior. Em $C$, cateterismo da artéria subclávia esquerda via femoral direita, observando-se a estenose do óstium da vertebral, a angulação do segmento inferior desta artéria, a bôa conirastação dos vasos intracranianos, além do enchimento, por refluxo, da artéria vertebral contralateral.

Vertebro-basilar angiography by netrograde catheterizxtion.

The three tipes of techniques for neuro-radiological investigation of the vertebro-basilar system — direct punction injection, counter-current injection and vascular catheterization - are revised and discussed.

The authors preconize the study of the vertebral-basilar system by the catheterization using the Seldinger's technic to which they attribute the following advantages: possibility of observation of the system from the 
subclavian artery up to the thiner vascular ramifications; absence of spams and artifacts commonly observed at direct punctions; facility of execution in routine examination. In patients with healthy vessels they introduce the catheter in the vertebral artery. In cases of vasculopathies they make the injection at the level of implantation of the a. vertebral in the a. subclavia. The authors believe that the catheterization is the more efficient way for neuro-radiological investigation of the vertebro-basilar system.

\title{
R E F E R E N I A S
}

1. BONNAL, J. \& LEGRE, J. - L'Angiographie cérebrale. Masson et Cie., Paris, 1958. 2. DAVID, M. - Traité de technique chirurgicale, Tome III, ler Fascicule, Masson et Cia., Paris, 1961. 3. HOWIESON, J. \& MEGISON JR., L. C. - Complications of vertebral catheterization. Radiology 91:1109, 1968. 4. JAEGER, R. \& WHITELEY, W. - Cerebral angiography by intravascular intubation technic. Amer. J. Roentgen. 73:735, 1955. 5. LABRUNE, M. \& DEBRUN, G. L'artériographie vértebrale chez l'enfant. Son intérêt, ses limites. Ann. Radiol. 11:751, 1968. 6. LINDGREN, E. - Percutaneous angiography of vertebral artery. Acta Radiol. 31:161, 1949. 7. LINDGREN, E. - Another method of vertebral angiography. Acta Radiol. 46:256, 1956. 8. LINDGREN, E. - Percutaneous angiography of vertebral artery. Acta Radiol. 33:398, 1956. 9. NEWTON, T. H.; KRAMER, R. A. \& MANI, J. R. - Catheter technic in vertebral arteriography. Radiology 87:691, 1966. 10. POUYANNE, H.; CAILLON, P.; LEMAN, P.; GOT, M.; SALLES, M. \& GANAZÉ, A. - L'Angiographie vertebrale par voie sous-clavière sousclaviculaire. Neurochirurgia 3:35, 1960. 11. RADNER, S. - Vertebral angiographie by catheterization. Acta Radiol. suppl. 167, 1951. 12. SOLE-LLENAS, J. - Diagnóstico Neurorradiologico. Ediciones Toray S. A., Barcelona, 1967. 13. TAVERAS, J. M. \& WOOD, E. - Diagnostic Neuroradiology. Williams and Wilkins Co., Baltimore, 1964. 14. ZACLIS, J.; LONGO, P. H. \& CRUZ, O. R. - Intrathoracic hiperpressure in neuroradiology: results in cerebral panangiography and arteriography of thoracocervical region. J. Neuro surg. 21:1087, 1964.

$* \quad * \quad * \quad *$

\section{ANGIOGRAFIA VÉRTEBRO:BASILAR TRANS-OPERATÓRIA POR CATETERISMO RETRÓGRADO}

\author{
SÉRgIO F. RAUPP * \\ Francisco F. RAMOS**
}

Em outros trabalhos tivemos oportunidade de relatar nossa experiência com a angiografia trans-operatória no decorrer de intervençōes visando a angiomas cerebrais $1,2,4,5$. Revisamos então 7 casos nos quais êste procedimento, permitindo reconhecer com certeza o(s) vaso(s) nutridor(es), havia mostrado ser excelente meio para a localização do angioma e para a avaliação do sucesso da exérese. Porém, a falta de fístulas congênitas na dependência do sistema vértebro-basilar não nos permitira encarar o problema da angiografia trans-operatória dêste sistema. 
No presente trabalho que é como que uma seqüência dos anteriores apresentamos as radiografias que obtivemos no decurso de ato cirúrgico mediante a colocação prévia de um catéter de Odman-Ledin pela técnica de Seldinger, via femoral, na artéria vertebral esquerda; o seguimento radiológico nos permitiu controlar o desenrolar do ato operatório, até a constatação do sucesso cirúrgico (fig. $1,2,3,4$ ).

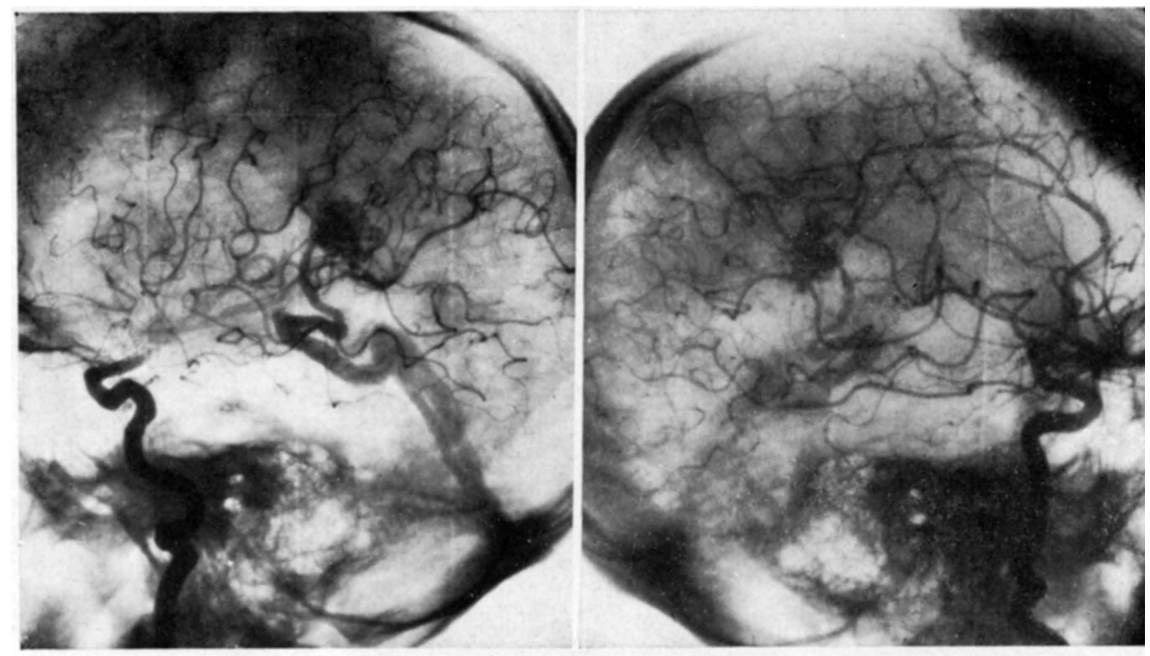

Fig. 1 - $\bar{A}$ esquerda, angiografia da carótida interna esquerda, por cateterismo seletivo, evidenciando fístula artério-venosa calosa posterior; à direita, angiografia da artéria carótida direita, por cateterismo seletivo.
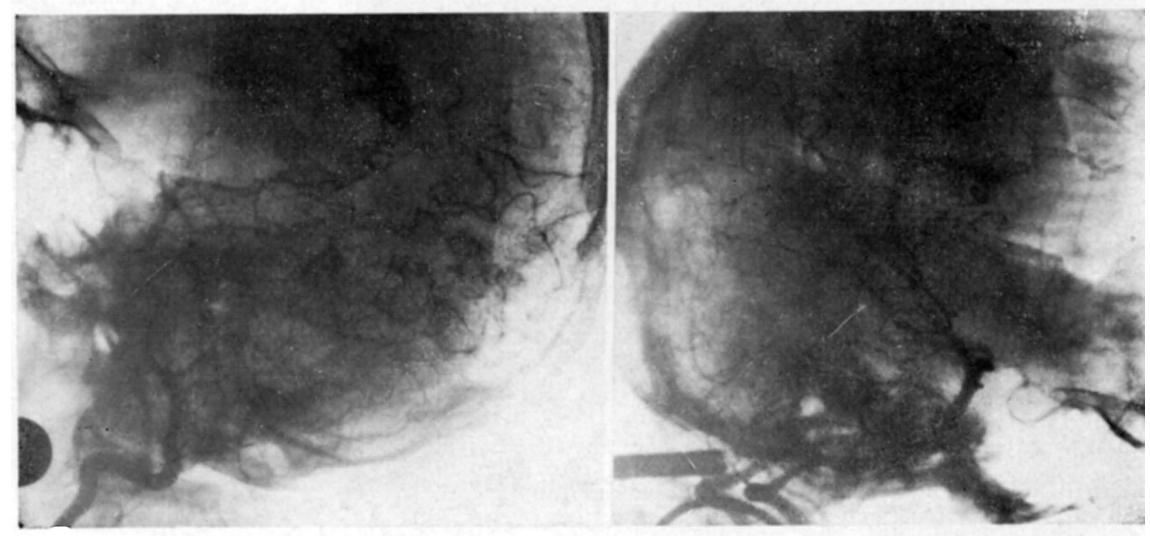

Fig. $2-\dot{A}$ esquerda, angiografia vértebro-basilar, por cateterismo seletivo, complementando a figura 1 e mostrando angioma caloso posterior, nutrido pelas artérias coroidéias e peri-calosas posteriores; à direita, angiografia vértebro-basilar trans-operatória, mostrando a exérese parcial da malformação. 

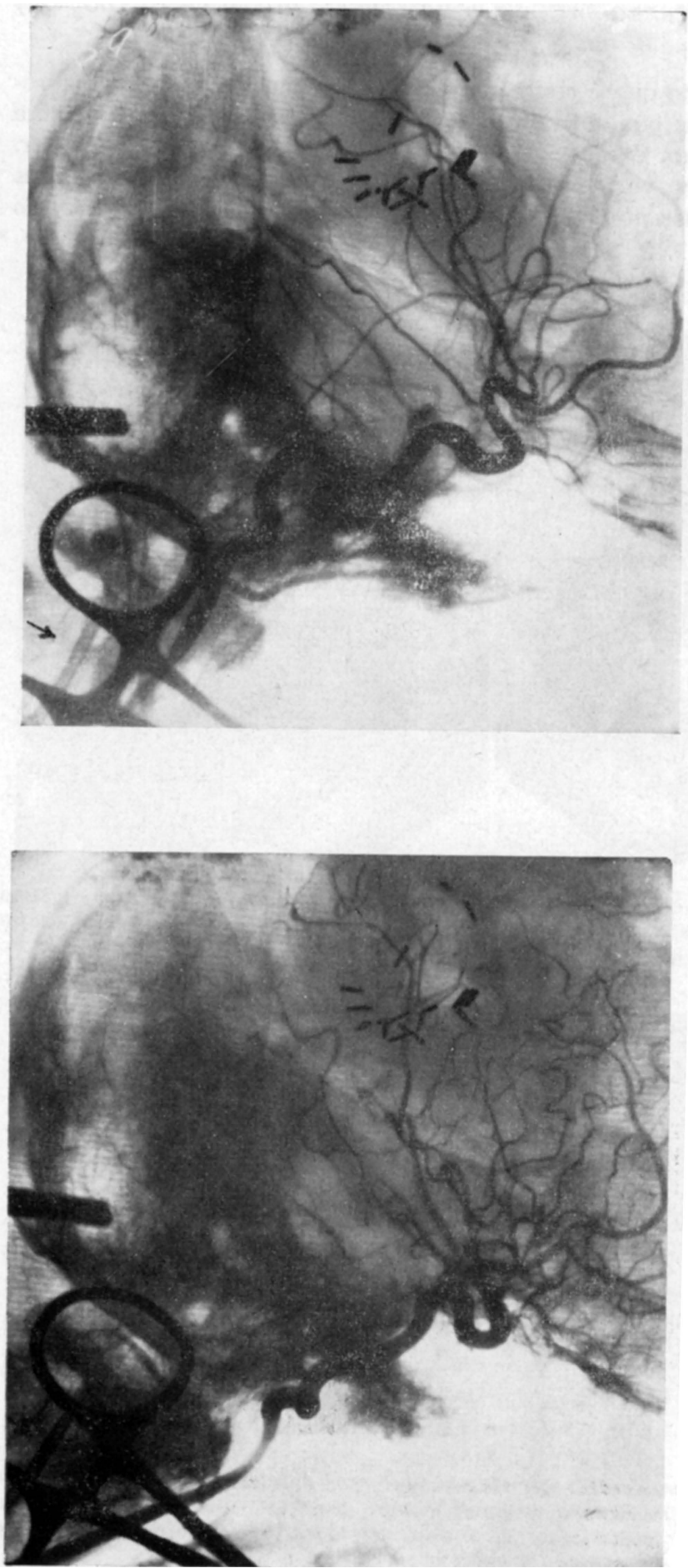

Fig. 3 - Angiografia da carótida direita, trans-operatória, mostrando estar completa a ligadura dos shunts de sua dependência, a seta indicando a localização do cateter radiopaco na artéria vertebral.

Fig. 4 - Angiografia da carótida esquerda, trans-operatória, mostrando não existir fistula na dependência desta artéria. 


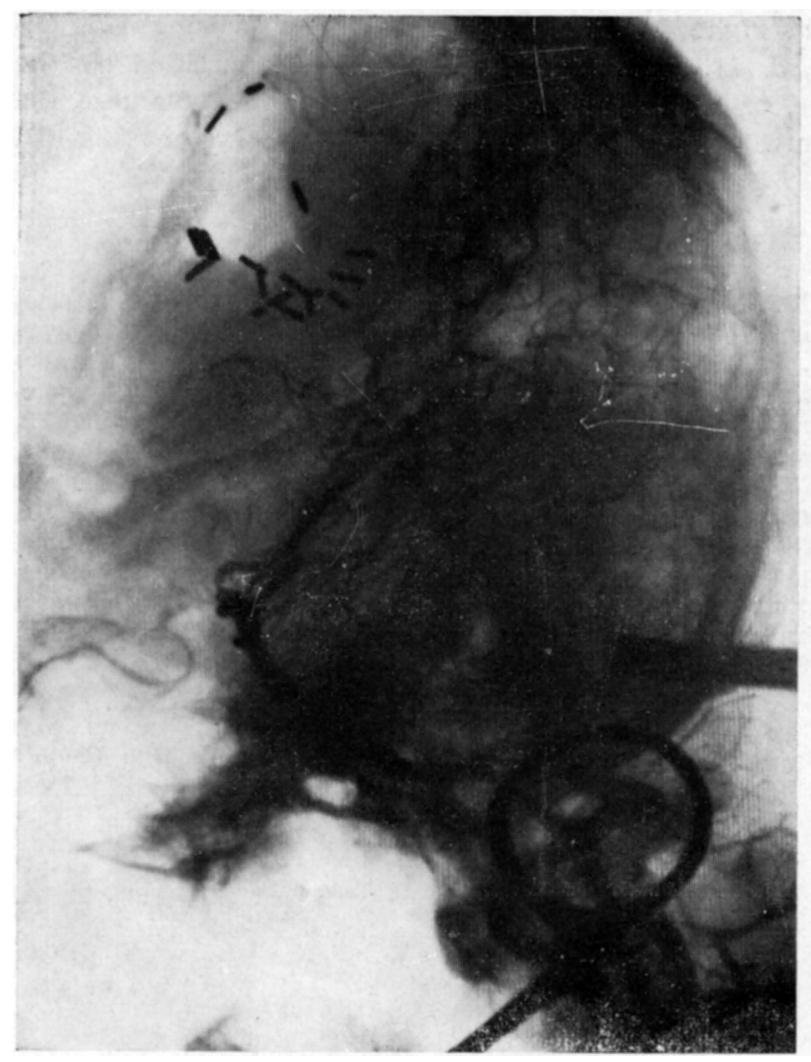

Fig. 5 - Angiografia vértebro-basilar, trans-operatória e final, mostrando ter sido feita exérese total da malformação.

O paciente, vítima de hemorragia cerebral, foi investigado por cateterismo seletivo de ambas as artérias carótidas internas e da artéria vertebral, sendo localizado angioma caloso posterior na dependência dos três sistemas.

A publicação do presente caso, como nota prévia, é justificada pela inexistência, na literatura ao nosso alcance, de relato da utilização do procedimento aqui referido com relação ao sistema vértebro-basilar ${ }^{3,6}$ e durante intervenção intracraniana.

\section{S U M M A R Y}

Vertebro-basilar angiography by retrograde catheterization during intracranial operation.

A case of an arteriovenous angioma supplied by the carotid and vertebro-basilar systems and located on the posterior half of the corpus callosum (fig. 1) is reported. The main source of blood flcw was given by the choroidal arteries and posterior callosal arteries (fig. 1a, 1b, 2a). During surgical operation the radiologic control was made by catheterization of the 
left vertebral artery, via right femoral artery (Seldinger), using Odman-Ledin catheter (fig. $2 b, 3,4$ ). The report of this case is justified by the absence, in the literature, of similar method to investigate and evaluate the success of surgical treatment in reference to vertebro-basilar system (fig. 5).

\section{R E F E R N C I A S}

1. DAHNE, J. \& RAUPP, S. - Tratamento cirúrgico das malformações artério-venosas do encéfalo. Atas do $\mathrm{V}$ Congresso da Sociedade Brasileira de Neurocirurgia, Belo Horizonte, 1964. 2. DAHNE, J. \& RAUPP, S. - Angiografia trans-operatória na cirurgia dos angiomas cerebrais. Atas do VI Congresso da Sociedade Brasileira de Neurocirurgia, São Paulo, 1966. 3. LOOF, W. J. \& FOLTZ, E. L. - Applications of anglography during intracranial operation. Acta Radiol. 5:363, 1966. 4. RAUPP, S. - Angiografia cerebral trans-operatória. Atas do VIII Congresso Nacional de Neurologia, Psiquiatria e Higiene Mental, Pôrto Alegre, 1967. 5. RAUPP, S.; TERUCHKIN, S. \& VIDAURRE, J. C. - Angiografia cerebral trans-operatória. Arq. Neuro-Psiquiat. (São Paulo) 26:141, 1968. 6. VLAHOVITCH, B.; FReREBEAU, Ph.; OUAKNINE, G.; BILlET, M. \& GROS, C. - Intérêt des angiographies per-opératoires dans les malformations vasculaires cèrebrales. Neuro-Chirurgie 15:191, 1969.

Instituto de Neurocirurgia - Hospital Săo Francisco - Pôrto Alegre, Rio Grande do Sul - Brasil. 